\title{
Objective Monitoring of Spectacle Wearing Times in Adult Subjects Using the Theramon ${ }^{\circledR}$ Thermosensor
}

Annegret Abaza (D)

Gideon Wahl'

Constanze Kortüm'

Kai Januschowski ${ }^{1,2}$

Dorothea Besch'

Charlotte Schramm'

'Department of Ophthalmology, Eberhard-Karls-University, Tuebingen, Germany; ${ }^{2}$ Department of Ophthalmology, Eye Clinic Sulzbach, Sulzbach, Saar, Germany
Correspondence: Annegret Abaza

Department of Ophthalmology, EberhardKarls-University, Elfriede-Aulhorn-Str. 7 ,

Tuebingen, 72076, Germany

$\mathrm{Tel}+49$ 707I-2988088

Fax +49 707I-293749

Email annegret.abaza@med.uni-

tuebingen.de
This article was published in the following Dove Press journal: Clinical Ophthalmology

Purpose: To find a reliable method to determine the wearing times of glasses in adults objectively on the basis of temperature measurements of the small-sized theramon ${ }^{\circledR}$ thermosensor device (TM) from orthodontics. Furthermore, the reliability of the determined wearing times during well and poor position of spectacles was explored. To address the previously reported lack of accuracy during higher outside temperatures, this study was conducted in midsummer and autumn.

Patients and Methods: Twenty adults wore their spectacles with three TMs attached to both inner sides and the right outer side in a period of three consecutive days. They were asked to report wearing and non-wearing times, indoor and outdoor activities and subjective perception of spectacle position as accurately as possible. To find the most reliable way to determine spectacle wearing times compared to protocolled wearing times, we applied and evaluated temperature categorizing methods and visual analysis of time logged temperatures. Percent error and percent accuracy of each method were calculated. The methods mentioned were applied and evaluated to determine poor spectacle position.

Results: Median percent errors of the most reliable determination methods were $<10 \%$ with an accuracy between $90 \%$ and $96 \%$. Comparing the most reliable methods, determinations on the basis of midsummer measurements (median percent error $0 \%$ to $2 \%$, median percent accuracy 96\%) were more accurate than on the basis of autumn measurements (median percent error $-5 \%$ to $-20 \%$, median percent accuracy $90 \%$ ). Poor spectacle position could be determined with a median percent error of $0 \%$ and a median percent accuracy of $100 \%$ by analysing the temperature measurements of TM's visually.

Conclusion: A reliable and objective determination of spectacle wearing times is possible on the basis of TM temperature measurements in a standard clinical setting. Measurements of one inner TM seem to be sufficient to determine the wearing times and are more appealing. Poor spectacle position can be determined reliably by analysing visually the time logged temperatures. The findings of this study require further studies on larger groups of affected, amblyopic patients and children, to analyse the real impact of spectacle wearing times and poor spectacle position on visual development.

Keywords: amblyopia, spectacle wearing times, objective monitoring, theramon ${ }^{\circledR}$ thermosensor, spectacle adherence

\section{Introduction}

Spectacle correction is an important part of the therapy of patients with different ophthalmic diseases. Adherence to spectacle wear is crucial, especially for children during the sensitive phase of visual development. ${ }^{1-3}$ The correction of refractive errors 
in children with anisometropic and/or strabismic amblyopia leads to an improvement of visual acuity in many cases ${ }^{4,5}$ and non-wearing of spectacles may have a negative impact on therapy success. Causes for non-adherence are variable, eg amount of uncorrected refractive error, level of uncorrected visual acuity or socio-demographic factors. ${ }^{6}$ At present, there is no established method to determine the spectacle wearing times reliably and objectively. Questionnaires for tracking wearing times may be utilized but their quality depends on the subjective notations and individual commitment of patients or parents.

To determine occlusion times in amblyopia-therapy objectively, thermosensors have been used since the early 1990s. The principle is, to determine occlusion times on the basis of logged temperatures in predefined time intervals. $^{7}$ Since their development, they have been constantly optimized and a lot of research has been done on different types of Occlusion-Dose-Monitors (ODM). ${ }^{7-12}$

Recent studies reported on different methods for determining spectacle wearing times by various thermosensor devices. One study investigated the adherence to spectacle wearing on 40 amblyopic children by using a GlassesDose-Monitor (GDM). The GDM is technologically similar to the ODM and measures temperature differences between two surfaces with a temperature resolution of $0.0625^{\circ} \mathrm{C}$. Data collection by GDM was successful in $85 \%$ and failed in around $15 \%$ due to technological problems or loss of monitors. The GDM is relatively large and must be attached with a patch to the spectacle frame. ${ }^{13}$ Another study reported on the SmartButton Data Logger Temperature Recorder, which was utilized to investigate spectacle wearing times of 50 adults objectively by Lentsch et al. This thermosensor can be easily mounted retroauricular to the end of the spectacle frame or above/ below it. Dependent on hairstyles and manner of fixation, measurements of the direct skin surface temperature were possible or not. Temperature readings were obtained with a resolution of $0.5^{\circ} \mathrm{C}$. Median percent error of determined spectacle wearing times was reported up to $8 \%$. Furthermore, determined wearing times were reported with a percent error of up to $10 \%$ for $64-70 \%$ of subjects and with a percent error of more than $20 \%$ for up to $26 \%$ of subjects. The largest percent errors were overestimations of spectacle wearing times. ${ }^{14}$

One recent study reported on the general applicability of the well-established theramon ${ }^{\circledR}$ thermosensor (TM) from orthodontics in ophthalmology. ${ }^{15,16}$ The small-sized sensor can be mounted to ocular patches and spectacle temples easily, which ensures direct contact to the wearer's skin surface. The results of this study show, that wearing $\mathrm{TM}$ in a pocket or placing it in an incubator was distinguishable from wearing it on spectacles or patches by $100 \%$ of the blinded test persons. A lack of accuracy was reported for temperatures of $33^{\circ} \mathrm{C}$ to $37^{\circ} \mathrm{C} .{ }^{17,18}$

The general purpose of this study was to evaluate and find further reliable methods to determine spectacle wearing times as well as poor spectacle positions objectively on the basis of measurements of the small-sized and widely available TM. To address the anciently reported lack of accuracy during higher outside temperatures, this study was conducted in midsummer and autumn.

\section{Methods}

\section{Subjects}

This prospective observational study was approved by the ethical committee of Eberhard-Karls-University Tübingen (reference number: 610/2014BO1) and adhered to the Declaration of Helsinki. All 20 adult subjects gave an informed consent.

\section{Criteria for Eligibility or Exclusion}

The requirement for the enrolment of subjects into the study was a spectacle correction of at least six hours/day. To enable the optimal attachment of TM, which means maintaining contact to the skin surface without applying uncomfortable pressure, a distance of around $4.6 \mathrm{~mm}$ between the subjects' spectacle frames and the temple area was required. That is $0.1 \mathrm{~mm}$ more than the TM height.

Adults with infrequent spectacle wearing (eg for driving or reading only), skin covering hair growth in the temple area or unsuitable styles of spectacle frames were excluded.

Each subject wore its spectacle with three TMs attached for three consecutive days and was asked to do its normal indoor and outdoor activities like sports, homework, gardening, driving, etc. Measurements were performed in midsummer (July/August) and autumn (October).

\section{Thermon ${ }^{\circledR}$ Thermosensor}

The TM has a compact format of $9 \times 13 \times 4.5 \mathrm{~mm}$. It is manufactured by MC Technology $\mathrm{GmbH}$ (Hargelsberg, Austria) and is commercially available worldwide. The flat side contains the thermosensor itself. The elevated rounded side contains the battery with a battery runtime of 15 months. With reading intervals of 15 minutes adjusted, the TM has 
a data storage of 100 days. Measurements have an accuracy of $\pm 0.2^{\circ} \mathrm{C}$. TM only wakes up to take measurements and remains in sleep mode during intervals in between. The data transfer from the TM to the computer works by a separately provided hardware, the TM reading station, in combination with a TM computer software. The connection between the $\mathrm{TM}$ and its reading station is based on the so-called radio frequency identification technology (RFID). The reading station itself is connected to the computer by USB. Temperature data can be exported from the TM software to Microsoft Excel.

\section{Attachment of the Theramon ${ }^{\circledR}$ Thermosensors}

A member of the study group attached three TM's to each subject's spectacles and verified their right location and proper fixation. The TM's were attached with their elevated rounded side to the spectacle frames by a doublesided strip (Figure 1). Two TMs were attached to both inner sides of the spectacle frames. Both were located preauricular at the height of the temporal region and in an area of minimal hair growth. Direct contact to the skin surface was verified. The third TM was attached to the right outer side of the spectacle frame without having contact with the subjects' skin surface.

\section{Data Collection}

All subjects were comprehensively informed about and fully aware of the automatic temperature recording by the TM. Furthermore, they were asked to keep accurate records in a standardised protocol, which required information about spectacle wearing and non-wearing times,

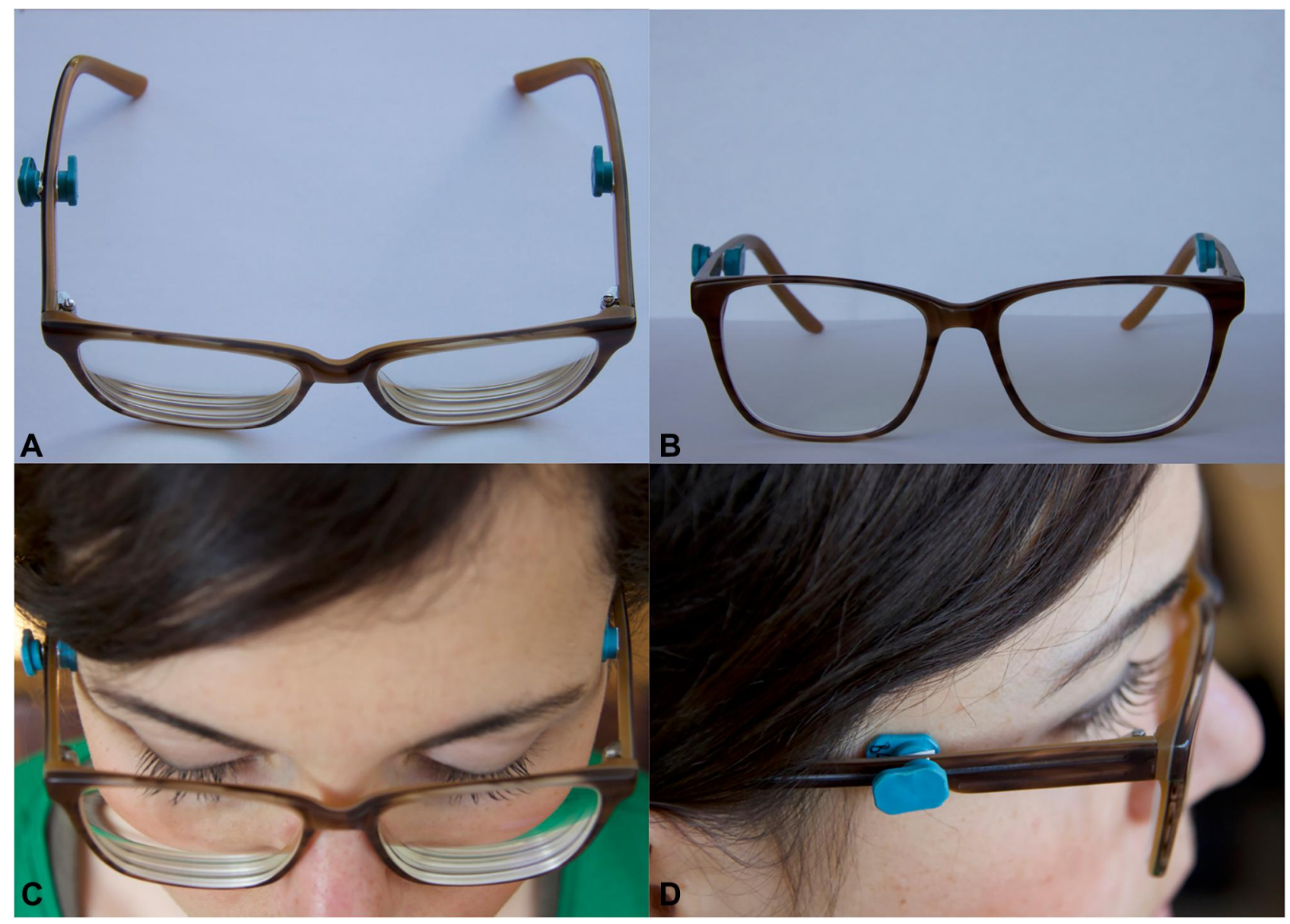

Figure I (A and B) Spectacle frames with three attached TM's. (C and D) Attachment of two TM's to both inner sides of the spectacle frame located preauricular at the height of the temporal region with direct contact to the skin surface. The third TM was attached to the right outer side of the spectacle frame without having contact to the subjects' skin surface.

Abbreviation: TM, theramon ${ }^{\circledR}$ thermosensor. 
indoor and outdoor activities with corresponding times, attached TM's and the subjective perception of the special events or environmental factors (eg sweating and spectacle position (see Figure 2 as an example of winding), the comfort of wearing spectacles with the a protocol).

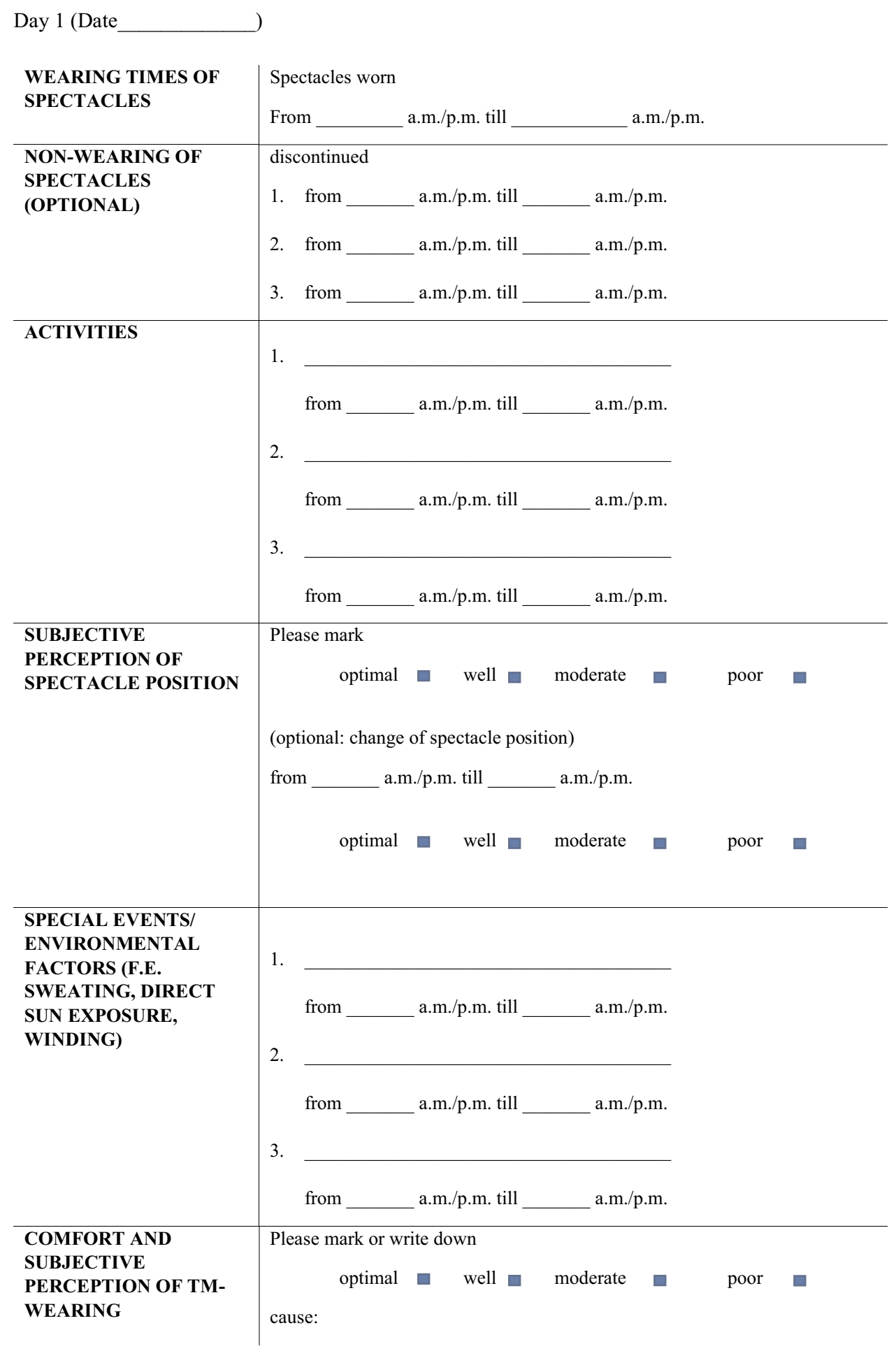

Figure 2 Example of the subjects' protocol.

Abbreviation: TM, theramon ${ }^{\circledR}$ thermosensor. 
Both, sensors and subjects' protocols were collected after 3 days by the study members and the temperature data of all TMs were transferred to Microsoft Excel.

\section{Statistical Analysis}

Export of Temperature Data and Protocolled Wearing Times

Temperature data were exported from the TM software to a Microsoft Excel file. The subjects' protocol data were transferred and saved in the same file for subsequent analysis, eg correlation with the temperatures measured by TMs.

\section{Correlating Theramon ${ }^{\circledR}$ Temperature Data with a Reference}

The temperatures measured by TMs were classified as "worn" and "not worn" according to the subjects' protocols. The subjects' protocol data were stated as truth: The protocolled wearing or non-wearing times in the subjects' protocols were defined as true "worn", respectively, true "not worn". The determined wearing or non-wearing times that did not match with the subjects' protocol data were defined as false "worn" and false "not worn". Group means and standard deviations (SD) of "worn" and "not worn" temperatures were calculated for each TM as well as for the calculated temperature differences between the TM's. The calculations were used for categorization of temperature data and determination of spectacle wearing times as described in the following.

\section{Definition of the Data for Determination Methods of Spectacle Wearing Times}

To determine spectacle wearing times with the methods described below, we used the temperature data measured by each TM as well as the calculated temperature differences between the TM's (hereinafter called determination data). For all determination data determination methods for spectacle wearing times were applied.

\section{Determination of Spectacle Wearing by Categorization of Temperature Data}

We used two different methods to determine wearing times. Both attempted to categorize the determination data into "worn" and "not worn" by cut-off-points. For method one, the temperature cut-off-point was calculated by the group mean of the protocolled "worn"-temperatures minus 1 SD. For method two it was calculated by the group mean of protocolled "not worn"-temperatures plus 1 SD.
Measured temperatures falling above/below these cutoff-points were stated as "worn"/“not worn" (Figure 3).

\section{Determination of Spectacle Wearing by Graphical Analysis of Temperature Data} We also investigated a visual determination method. Therefore, a blinded examiner tried to classify the determination data into "worn"/"not worn" by visually analysing the time-plotted determination data. A sudden steep increase in temperatures indicated that spectacles were put on. A subsequent steep decrease indicated that spectacles were taken off. In between temperatures oscillated slightly (Figure 4). Increase and decrease of temperatures were marked and spectacles were stated "worn" for the interval in between. Wearing times were calculated by counting the "worn" times (Figure 3).

\section{Comparison of the Determination Methods}

First, the percent error for each method was calculated using the following formula:

$$
\text { percent error }=\left(\frac{\begin{array}{l}
\text { determined } \\
\text { 'worn'time }
\end{array}-\begin{array}{c}
\text { protocolled } \\
\text { worn'time }
\end{array}}{\text { protocolled 'worn'time }}\right) \times 100
$$

Because the overall determined wearing times may be randomly similar to the overall wearing times in the subjects' protocols, we also calculated the percent accuracy for analysing more precisely the accuracy of the particular determination method:

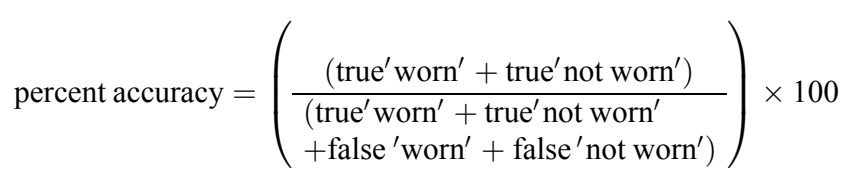

\section{Analysis of the Determinations for Poor Spectacle Position}

On the basis of the determination data and subjects' protocols, we determined the times of poor spectacle position or spectacle shifting by categorization of temperature data as described above. The percent error and percent accuracy of the determinations were calculated separately. Time plotted temperatures were analysed visually. 


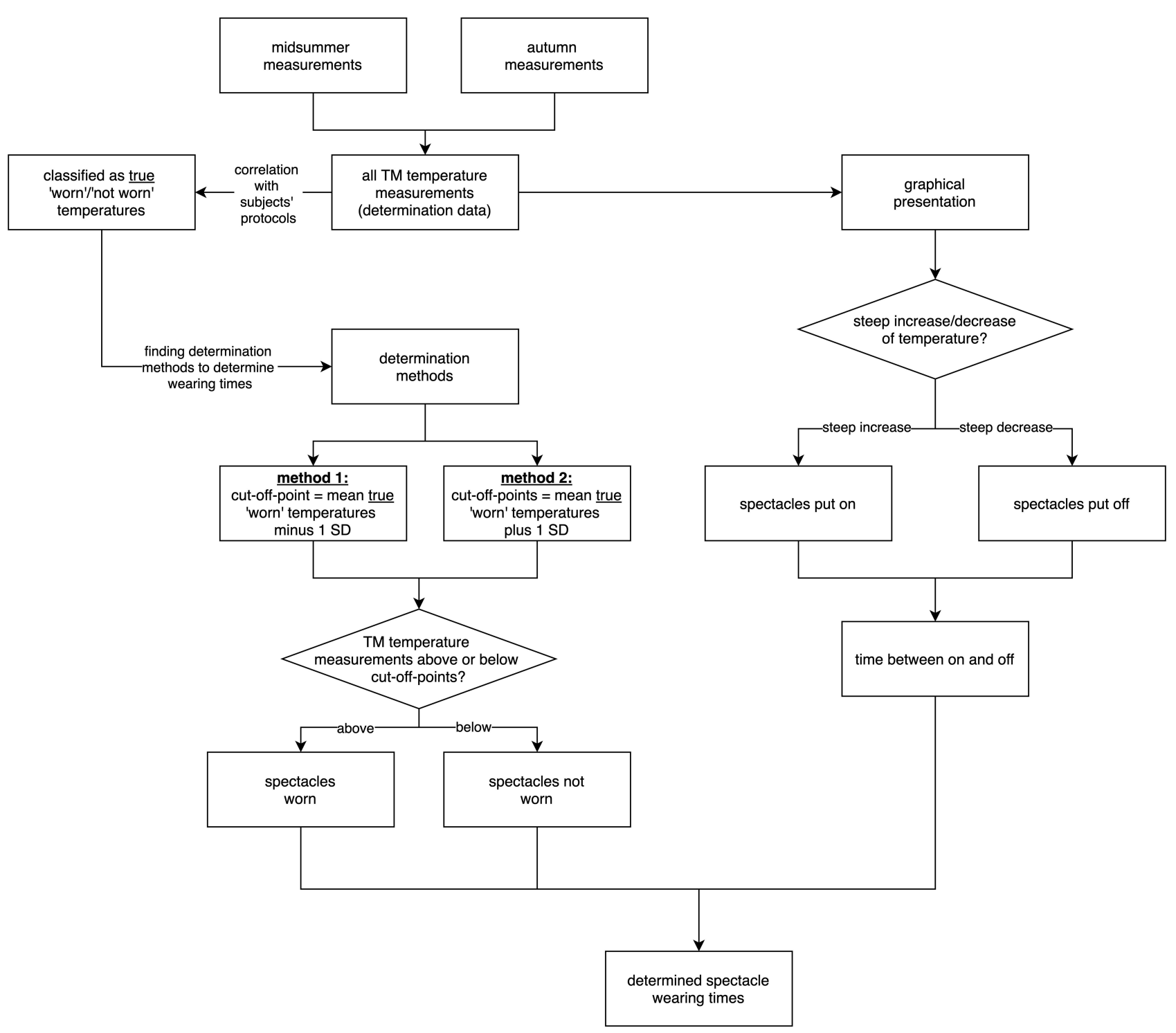

Figure 3 Flow diagram showing spectacle wearing groups and different methods of data analysis. Abbreviation: TM, theramon ${ }^{\circledR}$ thermosensor.

\section{Results}

All 20 subjects ( 12 female, 8 male) completed the 3 days of spectacle wearing with attached TM's and kept records of the data described above in the standardised subjects' protocols. All subjects were included in the analysis shown below.

The confidence of loggings was high: 19 subjects protocolled wearing and non-wearing times, indoor and outdoor activities and subjective spectacle position in detail during each day of TM measurements. One subject reported on wearing times but protocolled activities and spectacle position less detailed.

Three subjects reported discomfort due to pressure of the inner TM's on the temples. One subject reported a pressure of the inner TM's only while eating.
For two of the $60 \mathrm{TMs}$, the collection of temperature data was incomplete due to technological failure $(3.3 \%$ missed data storage, $96.7 \%$ successfully stored data). Both TMs were attached to the right outer side of the spectacles.

Temperature data were collected in midsummer (July/ August) and autumn (October). The outdoor temperature ranged between $17^{\circ} \mathrm{C}$ and $36^{\circ} \mathrm{C}$ in midsummer and $4^{\circ} \mathrm{C}$ and $13^{\circ} \mathrm{C}$ in autumn.

Group mean [SD] of "worn" and "not worn" temperatures for the determination data are shown in Table 1.

Mean temperatures of "worn" and "not worn" differed significantly for the right inner $\mathrm{TM}\left(\mathrm{F}_{1,18}=1971,6, \mathrm{p}<0.01\right)$, left inner TM $\left(\mathrm{F}_{1,18}=2473,3, \mathrm{p}<0.01\right)$, right outer TM $\left(\mathrm{F}_{1,16}\right.$ 


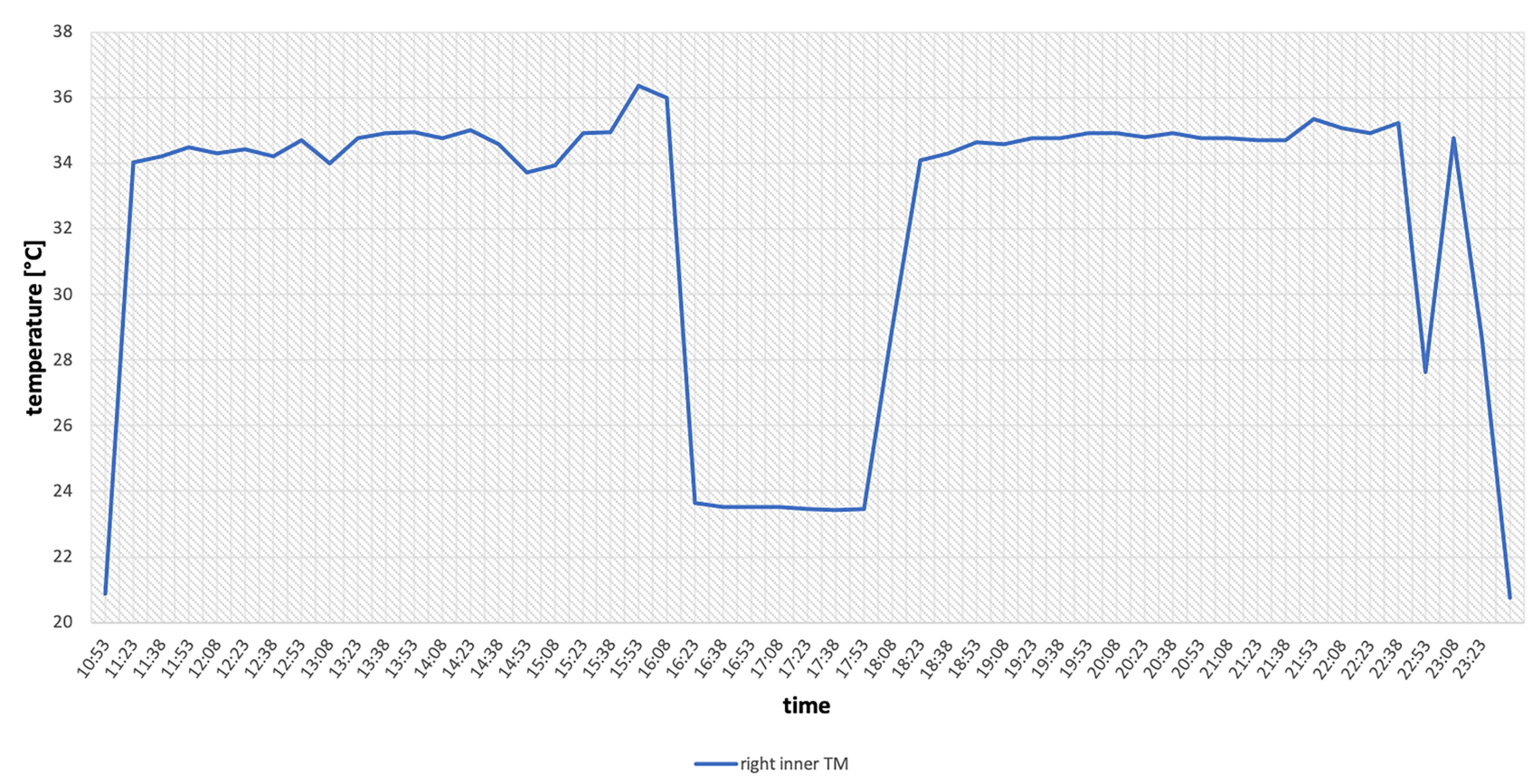

Figure 4 Time plotted temperature measurements of TM's $\left({ }^{\circ} \mathrm{C}\right)$. The steep increase and decrease of temperatures indicates that spectacles were put on or taken off, respectively. During the wearing times of spectacles temperature oscillates slightly. Abbreviation: TM, theramon ${ }^{\circledR}$ thermosensor.

$=264, \mathrm{p}<0.01), \Delta$ right inner TM-right outer TM $\left(\mathrm{F}_{1,16}=\right.$ $631,3, \mathrm{p}<0.01), \Delta$ left inner TM-right outer TM $\left(\mathrm{F}_{1,16}=\right.$ $965,5, \mathrm{p}<0.01)$ but not for the $\Delta$ right inner TM-left inner $\mathrm{TM}\left(\mathrm{F}_{1,18}=4.9, \mathrm{p}=0.02\right)$

The mean temperatures differed significantly between the gender for the right inner TM $\left(\mathrm{F}_{1,18}=16.4, \mathrm{p}<0.01\right)$, left inner TM $\left(\mathrm{F}_{1,18}=18.1, \mathrm{p}<0.01\right)$, right outer TM $\left(\mathrm{F}_{1,16}\right.$ $=78.3, \mathrm{p}<0.01), \Delta$ right inner TM-right outer $\mathrm{TM}\left(\mathrm{F}_{1,16}=\right.$ 49.8, $\mathrm{p}<0.01), \Delta$ left inner TM-right outer TM $\left(\mathrm{F}_{1,16}=\right.$ $57.9, \mathrm{p}<0.01)$ but not for the $\Delta$ right inner TM-left inner $\mathrm{TM}\left(\mathrm{F}_{1,18}=3.6, \mathrm{p}=0.05\right)$

In addition, the mean temperatures between the seasons differed significantly for the right inner TM $\left(\mathrm{F}_{1,18}=199,4\right.$, $\mathrm{p}<0.01)$, left inner TM $\left(\mathrm{F}_{1,18}=205,3, \mathrm{p}<0.01\right)$, right outer $\mathrm{TM}\left(\mathrm{F}_{1,16}=624, \mathrm{p}<0.01\right), \Delta$ right inner TM-right outer TM $\left(\mathrm{F}_{1,16}=320,3, \mathrm{p}<0.01\right), \Delta$ left inner TM-right outer TM
$\left(\mathrm{F}_{1,16}=378,7, \mathrm{p}<0.01\right)$ but not for the $\Delta$ right inner TM-left inner TM $\left(\mathrm{F}_{1,18}=2.7, \mathrm{p}=0.1\right)$.

Therefore, the temperature data of the $\Delta$ right inner TM-left inner TM were not included in the further statistical analysis.

\section{Wearing Times}

Group mean of protocolled daily spectacle wearing times was 10.7 hours ( $\mathrm{SD}=3.4$ hours). In comparison, group means of the determined wearing times are shown in Figure 5A.

Group means of protocolled daily spectacle wearing times were 10.6 hours ( $\mathrm{SD}=3.4$ hours) during midsummer and 9.5 hours ( $\mathrm{SD}=3.9$ hours) during the autumn. In comparison, the corresponding group means of our determinations are shown in Figure $5 \mathrm{~B}$ and $\mathrm{C}$.

Table I Group Means [I Standard Deviation] of TM Temperatures $\left({ }^{\circ} \mathrm{C}\right)$ Classified by Subjects' Protocols

\begin{tabular}{|l|c|c|c|c|c|c|}
\hline & $\begin{array}{c}\text { Right } \\
\text { Inner TM }\end{array}$ & $\begin{array}{c}\text { Left } \\
\text { Inner TM }\end{array}$ & $\begin{array}{c}\text { Right } \\
\text { Outer TM }\end{array}$ & $\begin{array}{c}\Delta \text { Right Inner TM-Left } \\
\text { Inner TM }\end{array}$ & $\begin{array}{c}\Delta \text { Right Inner TM-Right } \\
\text { Outer TM }\end{array}$ & $\begin{array}{c}\Delta \text { Left Inner TM-Right } \\
\text { Outer TM }\end{array}$ \\
\hline Worn & $33,3[2,4]$ & $33, I[2,4]$ & $28,3[3,6]$ & $0,1[1,9]$ & $4,9[2,5]$ & $4,9[2,4]$ \\
\hline Not Worn & $26,9[4,7]$ & $26, I[4,2]$ & $25,0[3,6]$ & $0,3[3,9]$ & $1,7[3,0]$ & $0,9[2,2]$ \\
\hline
\end{tabular}

Abbreviation: TM, theramon ${ }^{\circledR}$ thermosensor. 


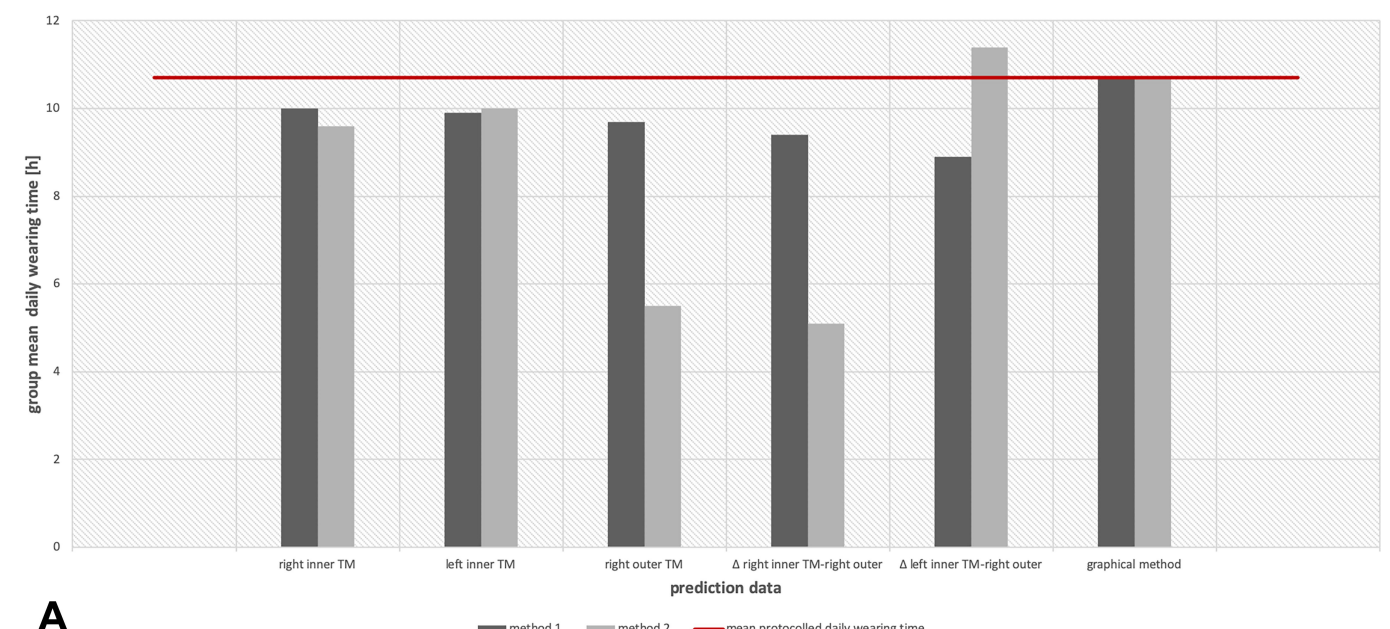

A

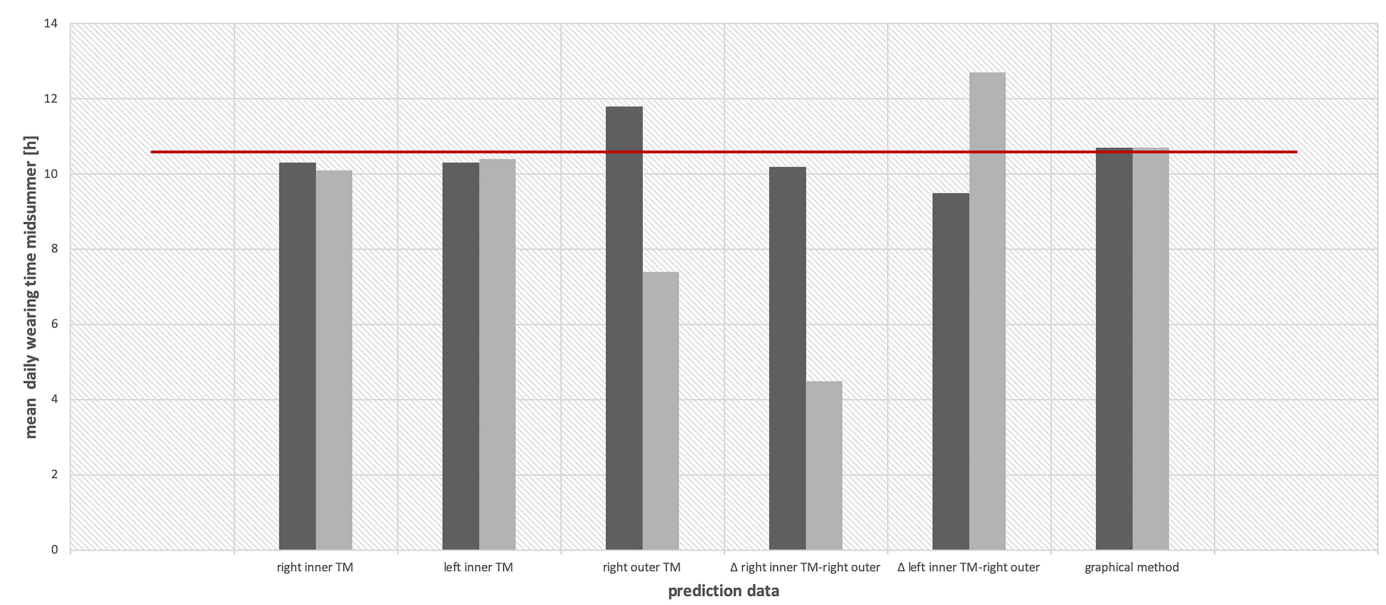

B

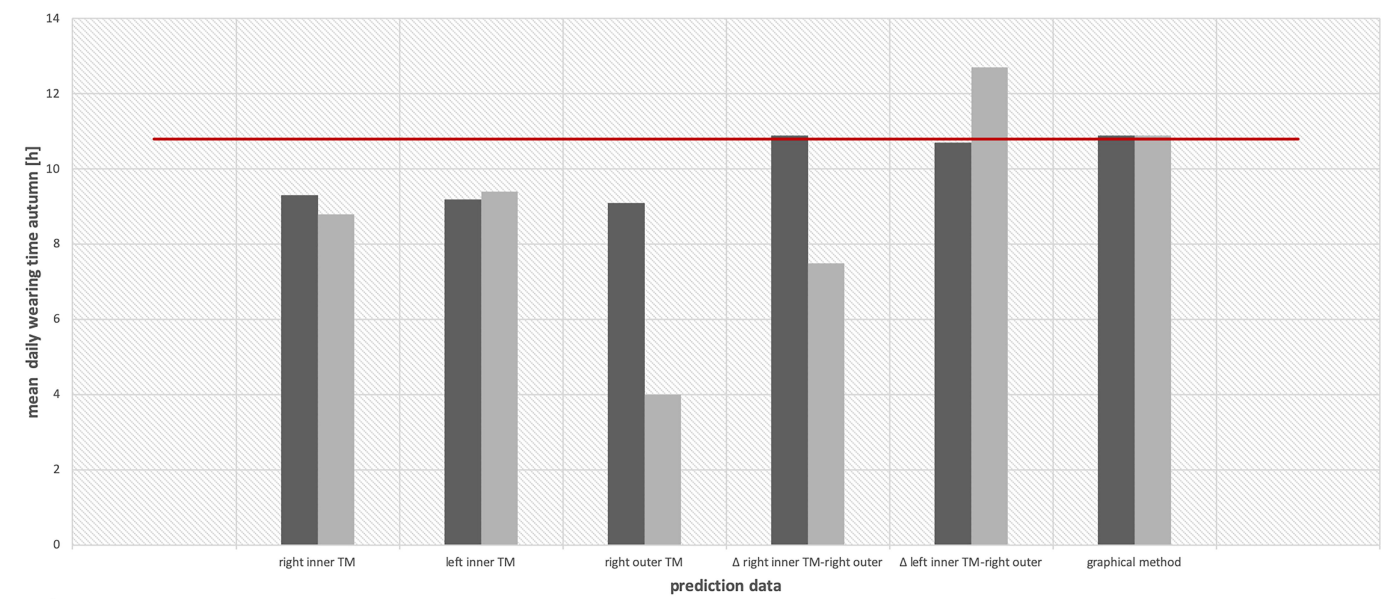

C

Figure 5 Means of determined daily wearing times (hours). (A) Group. (B) Midsummer group. (C) Autumn group. Abbreviations: h, hours; TM, theramon ${ }^{\circledR}$ thermosensor. 


\section{Comparison of the Determination Methods}

For all determination methods the distribution of percent error differed across the determination data sources $\left(\chi^{2}(10, \mathrm{~N}=208)\right.$

$=84.8, \mathrm{p}<0.001)$. There was a significant difference of percent error between the methods for right inner TM $\left(\mathrm{p}_{\text {method2-graphical method }}=0,0006\right)$, right outer TM ( $\mathrm{p}_{\text {method1- }}$ method2 $\left.=0.0012, \mathrm{p}_{\text {method2-graphical method }}=0.0005\right), \Delta$ right inner TM-right outer TM ( $\mathrm{p}_{\text {method1-method2 }}<0.0001, \mathrm{p}_{\text {method2- }}$ graphical method $<0.0001), \Delta$ left inner TM-right outer TM ( $\left.\mathrm{p}_{\text {method1-method2 }}<0.0001, \mathrm{p}_{\text {method2-graphical method }}<0.0001\right)$.

For all determination methods, there was no significant difference between the median percent errors across the seasons (Kruskal-Wallis-Test, $\mathrm{H}=10.8, d f=5, \mathrm{p}=0.05$ ) (Table 2).

The distribution of percent accuracy differed for all determination methods across the determination data sources $\left(\chi^{2}(10, \mathrm{~N}=208)=64.4, \mathrm{p}<0.001\right)$. There was a significant difference of percent accuracy between the determination methods for the right inner TM ( $\mathrm{p}_{\text {method1- }}$ grapical method $\left.=0.005, \mathrm{p}_{\text {method2-graphical method }}=0.0006\right)$, right outer $\mathrm{TM}$ ( $\mathrm{p}_{\text {method1-method2 }}=0.001, \mathrm{p}_{\text {method1-grapical }}$ method $\left.<0.0001, \mathrm{p}_{\text {method2-graphical method }}<0.0001\right), \Delta$ right inner TM-right outer TM ( $\mathrm{p}_{\text {method1-method2 }}<0.0001$, pmethod2-graphical method $<0.0001)$.

Across all determination methods the median percent accuracy differed significantly between midsummer and autumn (Kruskal-Wallis-Test, $\mathrm{H}=30.4, d f=5, \mathrm{p}<$ 0.001 ), but there was no difference of the same determination methods between the seasons $\left(\mathrm{p}_{\text {method } 1}=0.38\right.$; $\left.\mathrm{p}_{\text {method } 2}=0.68 ; \mathrm{p}_{\text {graphical method }}=0.81\right)($ Table 2).

The best determinations could be shown for determination methods 1 and 2 based on measurements of the left inner TM as well as for the graphical determination method. For method 1 the percent errors ranged between $-55 \%$ and $16 \%$ and the percent accuracy ranged between $44 \%$ and $99 \%$. For method 2 the percent errors ranged between $-53 \%$ and $19 \%$ and the percent accuracy ranged between $45 \%$ and $98 \%$. The percent error of determinations by graphical method ranged between $-6 \%$ and $7 \%$ and the percent accuracy between $91 \%$ and $98 \%$.

Determinations by method 2 based on the determination data of the right outer TM and $\Delta$ right inner TM-right outer TM were highest in median percent errors and lowest in median percent accuracy.
The highest percent errors of $-99 \%$ were shown for determination method 2 based on the determination data of the $\Delta$ right inner TM-right outer TM. The corresponding subject spent a lot of the time walking outside during high temperatures of around $30^{\circ} \mathrm{C}$. However, the determinations by method 2 based on the data of the right inner TM had a percent error of only $-4 \%$ for the same subject. Negative percent errors indicate an underestimation of spectacle wearing times.

Exemplary, time logged temperature measurements by the TM's are shown for subject 2 (Figure 6A). For the inner TM's, the increase and decrease of temperatures are similar. Simultaneously, for the right outer TM, there is a temperature increase/decrease at a lower level, which suggests the affection of measurements of the outer TM by the body temperature.

\section{Spectacle Position}

For protocolled optimal as well as for poor subjective spectacle position the time logged temperatures are shown exemplary for the right inner TM. For protocolled optimal position slight temperature oscillations can be detected (Figure 6B). For poor spectacle position or shifting of spectacles larger temperature oscillations can be detected (Figure 6C). Larger oscillations did not achieve the steep increase or decrease in measured temperatures when spectacles were put on or taken off.

The best determinations of wearing times during poor spectacle position were performed by determination method 1 on the basis of the temperature difference $\Delta$ left inner TM-right outer TM and the graphical analysis (Table 3).

\section{Discussion}

The results of this study show that wearing times of spectacles are objectively and reliably determinable by determination methods on the basis of the measurements of the TM's in a standard clinical setting. The best determinations of wearing times with the highest accuracy of determinations were obtained by the graphical determination method. Furthermore, both temperature categorizing methods revealed high accuracy of determinations. Interestingly, the determinations were more accurate for the midsummer group than for the autumn group. Position or shifting of spectacles could be determined reliably by analysing the plotted time logged temperature data of TM visually. 


\begin{tabular}{|c|c|c|c|c|c|c|c|c|c|c|c|}
\hline 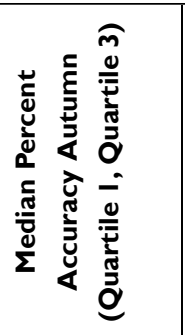 & 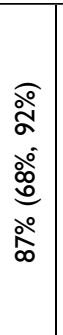 & 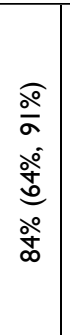 & 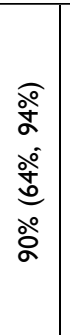 & 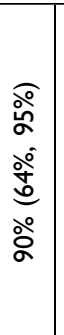 & 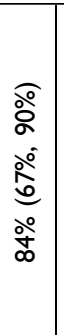 & 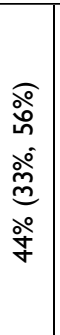 & 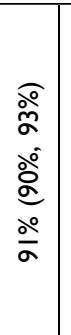 & 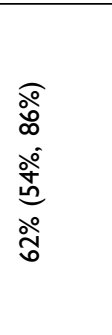 & 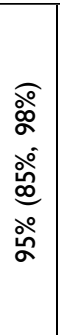 & 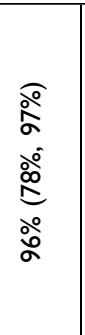 & 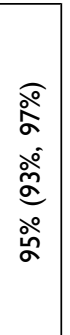 \\
\hline 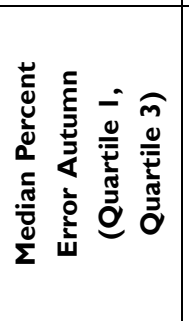 & 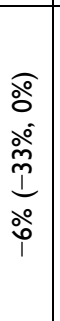 & 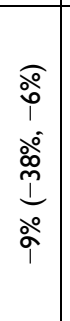 & 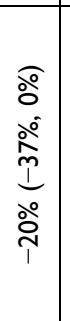 & $\begin{array}{l}\text { o } \\
\stackrel{\circ}{\circ} \\
\stackrel{\circ}{\tilde{P}} \\
\stackrel{\circ}{\circ}\end{array}$ & 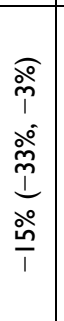 & 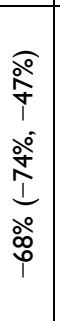 & $\begin{array}{c}\stackrel{\circ}{\circ} \\
\stackrel{0}{0} \\
\stackrel{0}{1} \\
\stackrel{0}{0}\end{array}$ & 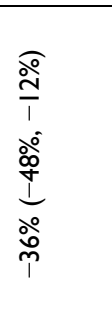 & 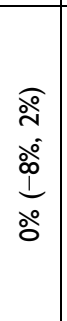 & 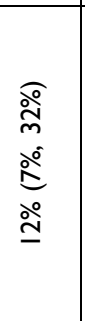 & 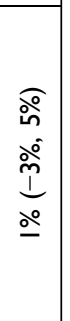 \\
\hline 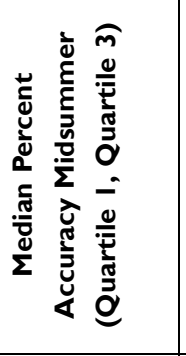 & 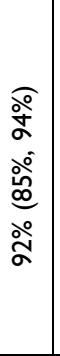 & 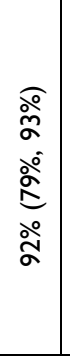 & 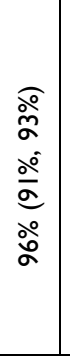 & $\begin{array}{l}\stackrel{\circ}{\circ} \\
\tilde{\sigma} \\
\frac{\circ}{\sigma} \\
\frac{0}{\circ} \\
\stackrel{\circ}{\circ}\end{array}$ & 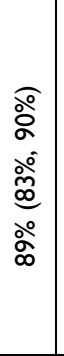 & 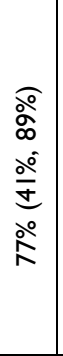 & 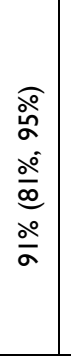 & 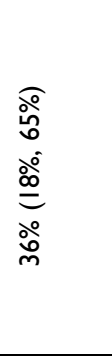 & 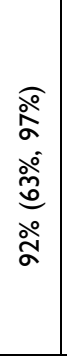 & $\begin{array}{l}\text { ळे } \\
\hat{\alpha} \\
\stackrel{\circ}{0} \\
\stackrel{0}{0} \\
\frac{\circ}{\infty}\end{array}$ & 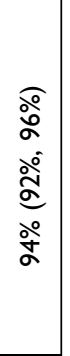 \\
\hline 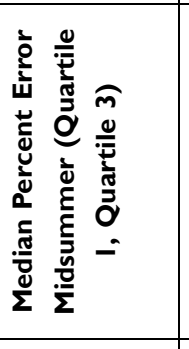 & 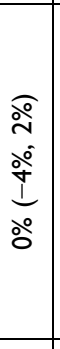 & $\begin{array}{c}\stackrel{\circ}{\circ} \\
\stackrel{\circ}{\circ} \\
\stackrel{1}{\circ} \\
\stackrel{0}{0}\end{array}$ & $\begin{array}{l}\stackrel{\circ}{\circ} \\
\stackrel{\circ}{0} \\
\stackrel{0}{0} \\
\stackrel{0}{0}\end{array}$ & 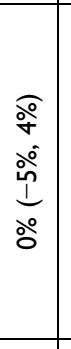 & 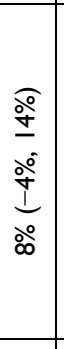 & 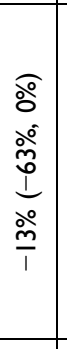 & 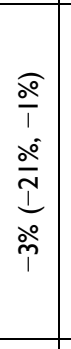 & 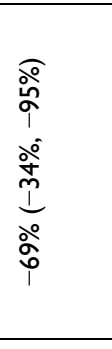 & 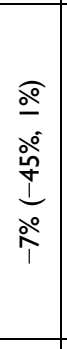 & $\begin{array}{l}\text { @े } \\
\stackrel{\circ}{\circ} \\
\stackrel{\circ}{\grave{0}}\end{array}$ & 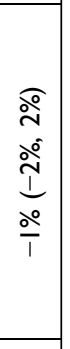 \\
\hline 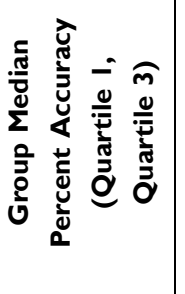 & 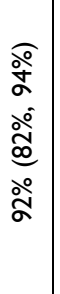 & 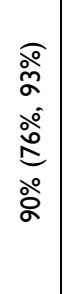 & 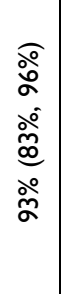 & 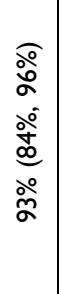 & $\begin{array}{l}\frac{0}{\sigma} \\
\frac{\partial}{\circ} \\
\frac{\partial}{\infty} \\
\stackrel{\circ}{\circ} \\
\infty\end{array}$ & 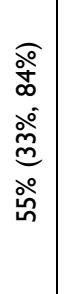 & $\begin{array}{l}\frac{\circ}{\grave{o}} \\
\frac{0}{\circ 0} \\
\frac{0}{\infty} \\
\frac{0}{\alpha}\end{array}$ & $\begin{array}{l}\text { ळo } \\
\stackrel{0}{\circ} \\
\stackrel{0}{0} \\
\stackrel{0}{0} \\
\stackrel{\circ}{\circ}\end{array}$ & 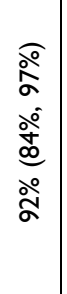 & 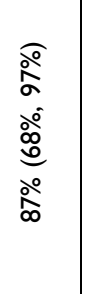 & 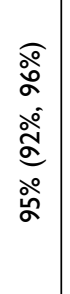 \\
\hline 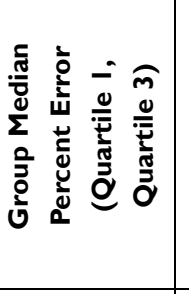 & 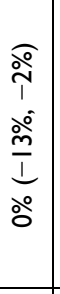 & $\begin{array}{c}\frac{0}{9} \\
\frac{0}{\circ} \\
\frac{0}{1} \\
\stackrel{0}{\circ}\end{array}$ & 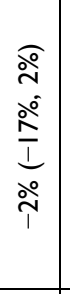 & $\begin{array}{c}\stackrel{\circ}{\circ} \\
\stackrel{\circ}{0} \\
\stackrel{0}{0} \\
\stackrel{0}{0}\end{array}$ & 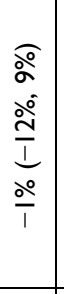 & 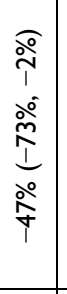 & $\begin{array}{l}\stackrel{\circ}{\circ} \\
\stackrel{\circ}{\circ} \\
\stackrel{0}{0} \\
\stackrel{0}{1}\end{array}$ & 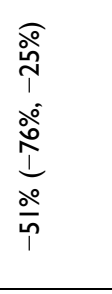 & $\begin{array}{l}\frac{\circ}{\circ} \\
\stackrel{\circ}{\stackrel{\circ}{1}} \\
\frac{\circ}{1}\end{array}$ & 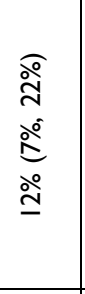 & 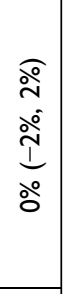 \\
\hline & \begin{tabular}{l|}
- \\
$\overline{0}$ \\
$\frac{c}{0}$ \\
$\frac{0}{\Sigma}$
\end{tabular} & 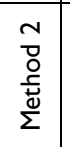 & $\begin{array}{l}\overline{-} \\
\overline{0} \\
\overline{0} \\
\overline{0} \\
\Sigma\end{array}$ & $\begin{array}{l}\text { N } \\
0 \\
0 \\
\bar{d} \\
\Sigma \\
\Sigma\end{array}$ & $\begin{array}{l}\bar{y} \\
\overline{0} \\
\frac{\bar{t}}{\Sigma} \\
\frac{\omega}{\Sigma}\end{array}$ & 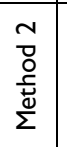 & $\begin{array}{l}\overline{-} \\
\overline{0} \\
\overline{0} \\
\overline{0} \\
\Sigma\end{array}$ & 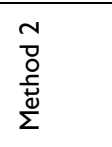 & $\begin{array}{l}\overline{-} \\
\overline{0} \\
\overline{0} \\
\overline{0} \\
\Sigma\end{array}$ & 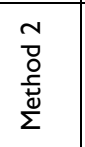 & \multirow{2}{*}{ 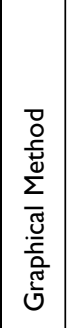 } \\
\hline & 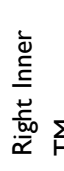 & & 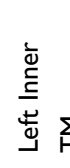 & & 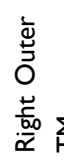 & & 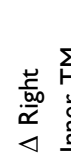 & 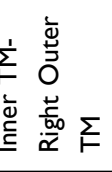 & & 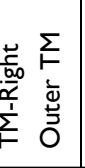 & \\
\hline
\end{tabular}



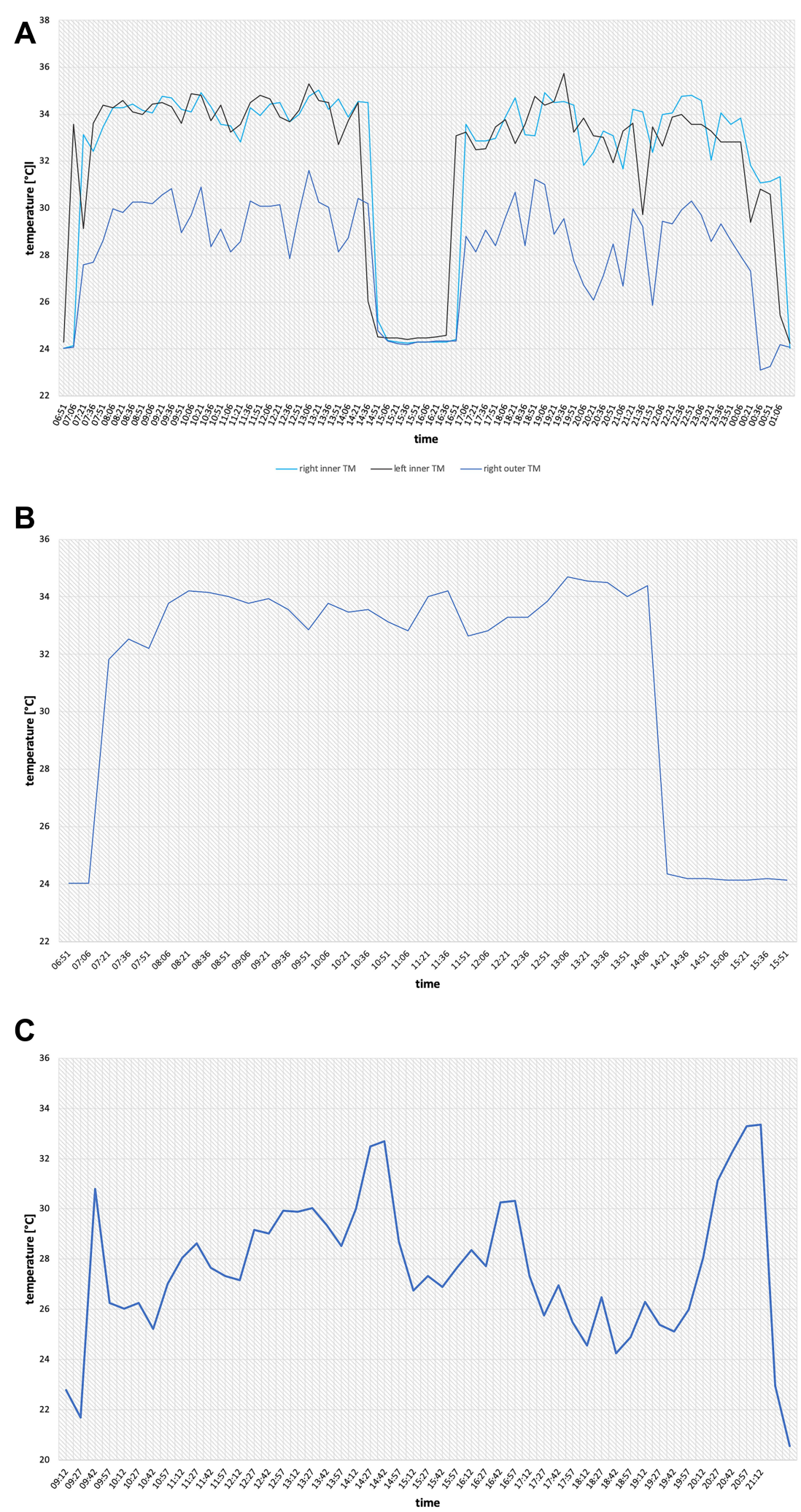

Figure 6 (A) Time plotted temperatures of both inner and the outer TM's $\left({ }^{\circ} \mathrm{C}\right)$ exemplary for subject 2. (B) Time plotted temperatures of right inner TM $\left({ }^{\circ} \mathrm{C}\right)$ during protocolled optimal spectacle position and (C) during protocolled poor spectacle position or shifting of spectacles.

Abbreviation: TM, theramon ${ }^{\circledR}$ thermosensor. 
Table 3 Median Percent Error and Median Percent Accuracy [Quartile I, Quartile 3] of Determined Wearing Times During Reported Poor Spectacle Position by Different Determination Methods.

\begin{tabular}{|c|c|c|c|}
\hline & & $\begin{array}{c}\text { Median Percent Error (Quartile I, } \\
\text { Quartile 3) }\end{array}$ & $\begin{array}{c}\text { Median Percent Accuracy (Quartile I, } \\
\text { Quartile 3) }\end{array}$ \\
\hline \multirow[t]{2}{*}{ Right Inner TM } & Method I & $0 \%(-50 \%, 0 \%)$ & $80 \%(50 \%, 100 \%)$ \\
\hline & Method 2 & $0 \%(-50 \%, 0 \%)$ & $80 \%(50 \%, 100 \%)$ \\
\hline \multirow[t]{2}{*}{ Left Inner TM } & Method I & $0 \%(-50 \%, 0 \%)$ & $100 \%(50 \%, 100 \%)$ \\
\hline & Method 2 & $0 \%(-50 \%, 0 \%)$ & $80 \%(50 \%, 100 \%)$ \\
\hline \multirow[t]{2}{*}{ Right Outer TM } & Method I & $-17 \%(-50 \%, 0 \%)$ & $80 \%(50 \%, 100 \%)$ \\
\hline & Method 2 & $-67 \%(-100 \%,-7 \%)$ & $33 \%(5 \%, 93 \%)$ \\
\hline \multirow{2}{*}{$\begin{array}{l}\Delta \text { Right Inner TM- Right Outer } \\
\text { TM }\end{array}$} & Method I & $0 \%(-25 \%, 6 \%)$ & $83 \%(75 \%, 100 \%)$ \\
\hline & Method 2 & $-19 \%(-83 \%, 0 \%)$ & $80 \%(17 \%, 95 \%)$ \\
\hline \multirow{2}{*}{$\begin{array}{l}\Delta \text { Left Inner TM-Right Outer } \\
\text { TM }\end{array}$} & Method I & $0 \%(-1 \%, 6 \%)$ & $99 \%(80 \%, 100 \%)$ \\
\hline & Method 2 & $0 \%(0 \%, 6 \%)$ & $90 \%(25 \%, 100 \%)$ \\
\hline \multicolumn{2}{|l|}{ Graphical Method } & $0 \%(0 \%, 0 \%)$ & $100 \%(80 \%, 100 \%)$ \\
\hline
\end{tabular}

Abbreviation: TM, theramon ${ }^{\circledR}$ thermosensor.

Even though the graphical method revealed the highest determination accuracy, we recommend the temperature categorizing method for the determination of spectacle wearing times in the clinical setting. Visual analysis of the plotted time logged temperature measurements is more time-consuming because the method is not automated yet and examiners must indicate increase and decrease of temperatures manually. In light of the evaluated methods of this study, it seems to be clinically more feasible to use the temperature categorizing methods. Also, for the temperature data of the inner TM's, those methods showed a high median determination accuracy of $>90 \%$ and a low median determination error of $<10 \%$.

In the future, the use of artificial intelligence for the determination of spectacle wearing times and spectacle position by the graphical method could be considered. For example, neuronal networks, methods of pattern recognition and deep learning could enable less timeconsuming and automated determinations with high accuracy. Probably, larger amounts of temperature data will be needed as test-data for the configuration and parametrization of the different algorithms of artificial intelligence. Afterwards, the methods of artificial intelligence could be employed for the temperature categorizing methods, too.

However, visually analysing the plotted temperature data of the TM's has advantages over the temperature categorizing methods for indicating spectacle position and shifting of spectacles. Large oscillations of temperatures indicate a poor spectacle position, whereas slight oscillations indicate that the spectacles were worn correctly.

An earlier TM study could show, that wearing the TM in a pocket can be distinguished from wearing it on spectacles or ocular patches with high accuracy by analysing temperature graphics, too. ${ }^{18}$ When TM was not in contact with the skin, temperature plateaus were detected and did not show the slight temperature oscillations, when spectacles or patches were worn.

In the clinical setting, it could be easy to evaluate the quality of the position of spectacles by the graphical method in addition to the temperature categorizing methods to determine the wearing times. Regarding the therapy of amblyopic children, a correct position of spectacles is of high importance for the best correction of refractive errors as well as for the therapy's success, especially in cases of higher amounts of astigmatism. A poor spectacle position or a spectacle shifting may lead to blurred vision and a negative impact on the development of visual acuity. In turn, adjustment of spectacles can be optimized easily by choosing suitable spectacle frames and additional straps or earpieces for children.

In addition, the temperature categorizing methods on the basis of the temperature differences between the left inner and the right outer TM determined the wearing and 
non-wearing times of spectacles with the highest accuracy and a low percent error in cases of poor spectacle position.

Surprisingly the results of this study indicate a difference between the determinations of the temperature categorizing methods on the basis of the temperature data of the right inner TM but not for the temperature data of the left inner TM. Differences might be caused by the small sample size of subjects as well as by the right outer TM, which possibly affected the temperature measurements of the right inner TM.

The determinations of wearing times on the basis of the temperature measurements of the outer TM showed the lowest accuracy. Therefore, we recommend not to use the outer TM in the clinical setting. Against our expectations, we could not show more accurate results for wearing time determinations on the basis of the temperature differences between the inner and outer TM, even in terms of different outside temperatures and seasons. In addition, the outer TM is more likely to lose due to its exposed localisation at the spectacle frame.

The different climatic conditions of seasons may affect the temperature measurements of thermosensor devices. Earlier thermosensor studies showed invalid measurements during very high outside temperatures over $33^{\circ} \mathrm{C} .{ }^{18,19}$

In this study, the median percent error for the temperature categorizing methods on the basis of the measurements of the inner TM's was lower in the midsummer group than in the autumn group. Also, the median percent accuracy was higher for determinations of the midsummer wearing times.

The results of this study are in concordance with the findings of Lentsch et al, who used the SmartButton Data Logger for monitoring of spectacle wearing of adults during warm and hot climatic conditions. They showed a low median percent error of $<10 \%$ for their approximations compared to the reported wearing times. ${ }^{14}$ Another study investigated the spectacle wearing times of adults for warm versus cold climate with the same SmartButton Data Logger. They applied the same temperature range, that was evaluated in the first study for warm climate, also for the cold climate and showed a median percent error of $4 \%$ in the summer and $-8 \%$ in the winter. ${ }^{20}$ Those findings are not unquestionable, because the subjects spent most of the time indoor.

In this study, we calculated and applied one temperature cut-off-point for both seasons on the basis of the group mean temperatures of the TM measurements. Compared to the results of Huang et al, one temperature range for different seasons seems not to be superior to compensate for the seasonal effect.
In conclusion, further investigations on monitoring spectacle wearing times by the TM during different seasons and extreme surrounding temperatures like very hot and very cold climates are required. Possibly, it is better to indicate seasonal cut-off-points adopted to the climate and outside temperatures.

In this study, subjects were not asked to wear their glasses full-time, because we aimed to evaluate temperature measurements of TM during different conditions of spectacle wearing and non-wearing. Regarding the therapy of amblyopic children, a full-time spectacle correction of 12 hours of daily wearing times is recommended, according to the sleep times of children. ${ }^{21}$ Therefore, it is important to consider the accuracy of the objectively determined spectacle wearing times for detection of adherence, because low adherence can be a cause of absent visual improvement. Maconachie et al could show with the measurements of the GDM, that median adherence to spectacle correction was only $70 \%$ and very variable in amblyopic children. ${ }^{13}$ A previous TM study on amblyopic children revealed an adherence to intermittent occlusion glasses, an alternative to occlusion patches, of only $51.6 \%{ }^{22}$

According to the results of the most accurate determination method (method 2 on the basis of the temperature data of the left inner TM), the determined wearing times would be 12 hours per day in midsummer (median percent error $0 \%$ ) and 11.4 hours during the autumn (median percent error - 5\%), which would indicate high adherence to spectacle wearing. Also, lower adherence can be detected reliably by the evaluated method of this study. Amblyopic children and their parents could be encouraged to the spectacle wearing by the visualized wearing times as positive feedback.

Most of the subjects in this study did not have any complaints while wearing their spectacles with the attached TM's. However, four subjects reported discomfort due to pressure of inner TM's on their temples. It is important to verify the suitability of the TM to the present spectacle frames to reduce the sense of pressure. Especially in terms of affected amblyopic children, acceptance and comfort must be tested.

Regarding the suitability to spectacle frames as well as aesthetical aspects, the TM's small size is of advantage compared to other thermosensor devices like the SmartButton Data logger ${ }^{14}$ or the GDM. ${ }^{13}$ These thermosensors are larger in size and need to be mounted to spectacle frames by more conspicuous tapes. Even though our subjects did not lose any of the TM's, one concern of the small size is the risk for loss while putting spectacles 
on or taking them off. In the clinical setting other mounting strategies could be tested.

This study has several limitations: All subjects were adults and measurements were obtained outside a therapeutic setting. The high confidence in subjects' protocols could be due to the fact that subjects participating in this study were particularly motivated. The high educational and socio-demographic level of subjects leads to a homogeneity of the group. Possibly measurements on affected amblyopic children of different sociodemographic levels may differ from our findings.

Cut-off-points for temperature categorizing methods may differ between seasons. Especially exposition to extreme hot or cold temperatures may have an impact on the measurements and the accuracy of the determinations. Therefore, it could be of advantage to identify individual cut-off-points adopted to the climatic conditions, even though in this study, the percent errors of the most accurate determination methods were shown to be $<10 \%$ and did not differ in accuracy between midsummer and autumn measurements.

\section{Conclusion}

The results of this study indicate that spectacle wearing times are objectively and reliably determinable on the basis of TM temperature measurements in a standard clinical setting. Determinations based on the measurements of one inner TM seem to be sufficient and more appealing. We recommend to determine wearing times by the automatable temperature categorizing method two. The quality of the spectacle position can be easily evaluated by visually analysing the plotted time logged temperatures.

The findings of this study require further studies on larger groups of affected, amblyopic patients and children, evaluating the real impact of refractive correction.

\section{Abbreviations}

$\mathrm{TM}$, theramon ${ }^{\circledR}$ thermosensor; SD, standard deviation.

\section{Data-Sharing Statement}

All data mentioned in the study are available and can be supplied upon request from the corresponding author.

\section{Ethics Approval}

All procedures performed in the study were in accordance with the ethical standards of the Institutional Review Board and ethical committee of Eberhard-KarlsUniversity Tübingen and with the 1964 Declaration of Helsinki and its later amendments.

\section{Informed Consent}

Informed consent to participate and for publication was obtained from all participants included in the study. The person in Figure 1 has provided informed consent for the image to be published.

\section{Funding}

No funding was received throughout the study.

\section{Disclosure}

The authors declare that they have no conflicts of interest for this work.

\section{References}

1. Hubel DH, Wiesel TN. The period of susceptibility to the physiological effects of unilateral eye closure in kittens. $J$ Physiol. 1970;206 (2):419-436. doi:10.1113/jphysiol.1970.sp009022

2. von Noorden GK. Amblyopia: a multidisciplinary approach. Proctor lecture. Invest Ophthalmol Vis Sci. 1985;26(12):1704-1716.

3. von Noorden GK, Crawford ML. The sensitive period. Trans Ophthalmol Soc U K. 1979;99(3):442-446.

4. Moseley MJ, Neufeld M, McCarry B, et al. Remediation of refractive amblyopia by optical correction alone. Ophthalmic Physiol Opt. 2002;22(4):296-299. doi:10.1046/j.1475-1313.2002.00034.x

5. Stewart CE, Moseley MJ, Fielder AR, et al. Refractive adaptation in amblyopia: quantification of effect and implications for practice. $\mathrm{Br}$ J Ophthalmol. 2004;88(12):1552-1556. doi:10.1136/bjo.2004.044214

6. Morjaria P, McCormick I, Gilbert C. Compliance and predictors of spectacle wear in schoolchildren and reasons for non-wear: a review of the literature. Ophthalmic Epidemiol. 2019;26(6):367-377. doi:10.1080/09286586.2019.1628282

7. Fielder AR, Irwin $M$, Auld $R$, et al. Compliance in amblyopia therapy: objective monitoring of occlusion. $\mathrm{Br} J$ Ophthalmol. 1995;79(6):585-589. doi:10.1136/bjo.79.6.585

8. Awan M, Proudlock FA, Gottlob I. A randomized controlled trial of unilateral strabismic and mixed amblyopia using occlusion dose monitors to record compliance. Invest Ophthalmol Vis Sci. 2005;46 (4):1435-1439. doi:10.1167/iovs.04-0971

9. Simonsz HJ, Polling JR, Voorn R, et al. Electronic monitoring of treatment compliance in patching for amblyopia. Strabismus. 1999;7 (2):113-123. doi:10.1076/stra.7.2.113.645

10. Stewart CE, Moseley MJ, Stephens DA, et al. Treatment dose-response in amblyopia therapy: the monitored occlusion treatment of amblyopia study (MOTAS). Invest Ophthalmol Vis Sci. 2004;45(9):3048-3054. doi:10.1167/iovs.04-0250

11. Chopovska Y, Loudon SE, Cirina L, et al. Electronic recording of occlusion treatment for amblyopia: potential of the new technology. Graefes Arch Clin Exp Ophthalmol. 2005;243(6):539-544. doi:10.1007/s00417-004-1067-8

12. Loudon SE, Polling JR, Simonsz HJ. Electronically measured compliance with occlusion therapy for amblyopia is related to visual acuity increase. Graefes Arch Clin Exp Ophthalmol. 2003;241 (3):176-180. doi:10.1007/s00417-002-0570-z

13. Maconachie GD, Farooq S, Bush G, et al. Association between adherence to glasses wearing during amblyopia treatment and improvement in visual acuity. JAMA Ophthalmol. 2016;134 (12):1347-1353. doi:10.1001/jamaophthalmol.2016.3793

14. Lentsch MJ, Marsack JD, Anderson HA. Objective measurement of spectacle wear with a temperature sensor data logger. Ophthalmic Physiol Opt. 2018;38(1):37-47. doi:10.1111/opo.12423 
15. Schott TC, Goz G. Applicative characteristics of new microelectronic sensors Smart Retainer(R) and TheraMon(R) for measuring wear time. J Orofac Orthop. 2010;71(5):339-347. doi:10.1007/s00056010-1019-3

16. Schott TC, Goz G. Wearing times of orthodontic devices as measured by the TheraMon(R) microsensor. J Orofac Orthop. 2011;72 (2):103-110. doi:10.1007/s00056-011-0014-7

17. Januschowski K, Rickmann A, Emmerich C, et al. Der Einsatz eines Mikrosensors in der Amblyopietherapie [The use of a microsensor in therapy of amblyopia]. Klin Monbl Augenheilkd. 2017;236:1170-1173. German.

18. Januschowski K, Bechtold TE, Schott TC, et al. Measuring wearing times of glasses and ocular patches using a thermosensor device from orthodontics. Acta Ophthalmol. 2013;91(8):e635-640. doi:10.1111/ aos. 12171
19. Fronius M, Chopovska Y, Nolden J, et al. Occlusion treatment for amblyopia: assessing the performance of the electronic occlusion dose monitor. Strabismus. 2006;14(2):65-70. doi:10.1080/ 09273970600700962

20. Huang J, Lentsch MJ, Marsack JD, et al. Evaluating the use of a temperature sensor to monitor spectacle compliance in warm versus cold climates. Clin Exp Optom. 2019;102(2):147-153. doi:10.1111/ cxo. 12843

21. Williams JA, Zimmerman FJ, Bell JF. Norms and trends of sleep time among US children and adolescents. JAMA Pediatr. 2013;167 (1):55-60. doi:10.1001/jamapediatrics.2013.423

22. Wang J, Jin J, Malik A, et al. Feasibility of monitoring compliance with intermittent occlusion therapy glasses for amblyopia treatment. $J \quad A A P O S$. 2019;23(4):205e201-205 e205. doi:10.1016/j. jaapos.2019.04.009
Clinical Ophthalmology

\section{Publish your work in this journal}

Clinical Ophthalmology is an international, peer-reviewed journal covering all subspecialties within ophthalmology. Key topics include: Optometry; Visual science; Pharmacology and drug therapy in eye diseases; Basic Sciences; Primary and Secondary eye care; Patient Safety and Quality of Care Improvements. This journal is indexed on PubMed

Submit your manuscript here: https://www.dovepress.com/clinical-ophthalmology-journal
Dovepress

Central and CAS, and is the official journal of The Society of Clinical Ophthalmology (SCO). The manuscript management system is completely online and includes a very quick and fair peer-review system, which is all easy to use. Visit http://www.dovepress.com/ testimonials.php to read real quotes from published authors. 